Supporting Information for submission to ACS Bioconjugate Chemistry

\title{
Human Granulocyte-Macrophage Colony-Stimulating Factor Fused to Elastin-Like Polypeptides Assembles Biologically-Active Nanoparticles
}

Mincheol Park ${ }^{\dagger}$, Vijaya P. Vaikari ${ }^{\ddagger}$, Jugal P. Dhandhukia ${ }^{\dagger}$, Houda Alachkar ${ }^{\ddagger}$, , J. Andrew MacKay*t,\|,

†Department of Pharmacology and Pharmaceutical Sciences, School of Pharmacy, University of Southern California, Los Angeles, California 90089, United States

‡Department of Clinical Pharmacy, School of Pharmacy, University of Southern California, Los Angeles, California 90089, United States

\&USC Norris Comprehensive Cancer Center, University of Southern California, Los Angeles, California 90089, United States

"Department of Ophthalmology, USC Roski Eye Institute, Keck School of Medicine, University of Southern California, Los Angeles, California 90089, United States

"Department of Biomedical Engineering, Viterbi School of Engineering, University of Southern California, Los Angeles, California 90089, United States

*Email: jamackay@usc.edu. Telephone: (323) 442-4118

Conflict of Interest Statement: Funding for this work was provided by the United States National Institutes of Health, the University of Southern California, and the L.K. Whittier Foundation. J.A.M, J.P.D., and M.P. are inventors on patents related to elastin-like polypeptides. J.A.M. holds equity in a limited liability company that seeks to commercialize an elastin-like polypeptide formulation. 


\section{Supporting Information for the purity and characteristic of hGMCSF-A192}

To measure the purity of hGMCSF-A192, copper-stained SDS-PAGE gel images of two different batches of hGMCSF-A192 were analyzed with ImageJ, and area under the curve (AUC) of the UV trace of hGMCSF-A192 from size-exclusion chromatography with multi-angle light scattering (SEC-MALS) was calculated. The UV trace of hGMCSF-A192 from SEC-MALS showed one major peak and one minor peak, and the AUC of two peaks was calculated. The AUC for the major peak and the minor peak was measured to be 0.4558 and $0.003559 \mathrm{~mL}$, respectively, and the purity of hGMCSF-A192 was determined to be $99.2 \%$ (Figure S1A). The purity of the first batch of hGMCSF-A192 measured from the copper-stained gel was $89.2 \%$ (Figure S1B). On the other hand, the purity of the second batch of hGMCSF-A192 measured from the copper-stained gel was close to $\sim 100 \%$ as no other peaks were observed on the gel (Figure 1D and S1C). Therefore, the purity of hGMCSF-A192 was determined to be $96.1 \pm 6.0 \%$ (mean \pm SD, $n=3$ ).

As hGMCSF-A192 forms two disulfide bonds, the fusion protein was run on an SDS-PAGE gel with and without reducing agent in order to verify whether hGMCSF-A192 forms disulfide bonds intermolecularly. When the fusion protein was run on the gel with a reducing agent, the protein band was observed near the $100 \mathrm{kDa}$ marker, which is close to the molecular weight of hGMCSFA192. On the other hand, when the fusion protein was run without a reducing agent, hGMCSFA192 was not able to run on the gel, indicating that the fusion protein forms disulfide bonds intermolecularly (Figure S1C). This observation supports that the hGMCSF domains form the core of the hGMCSF-A192 nanoparticles by forming some disulfide bonds intermolecularly. 


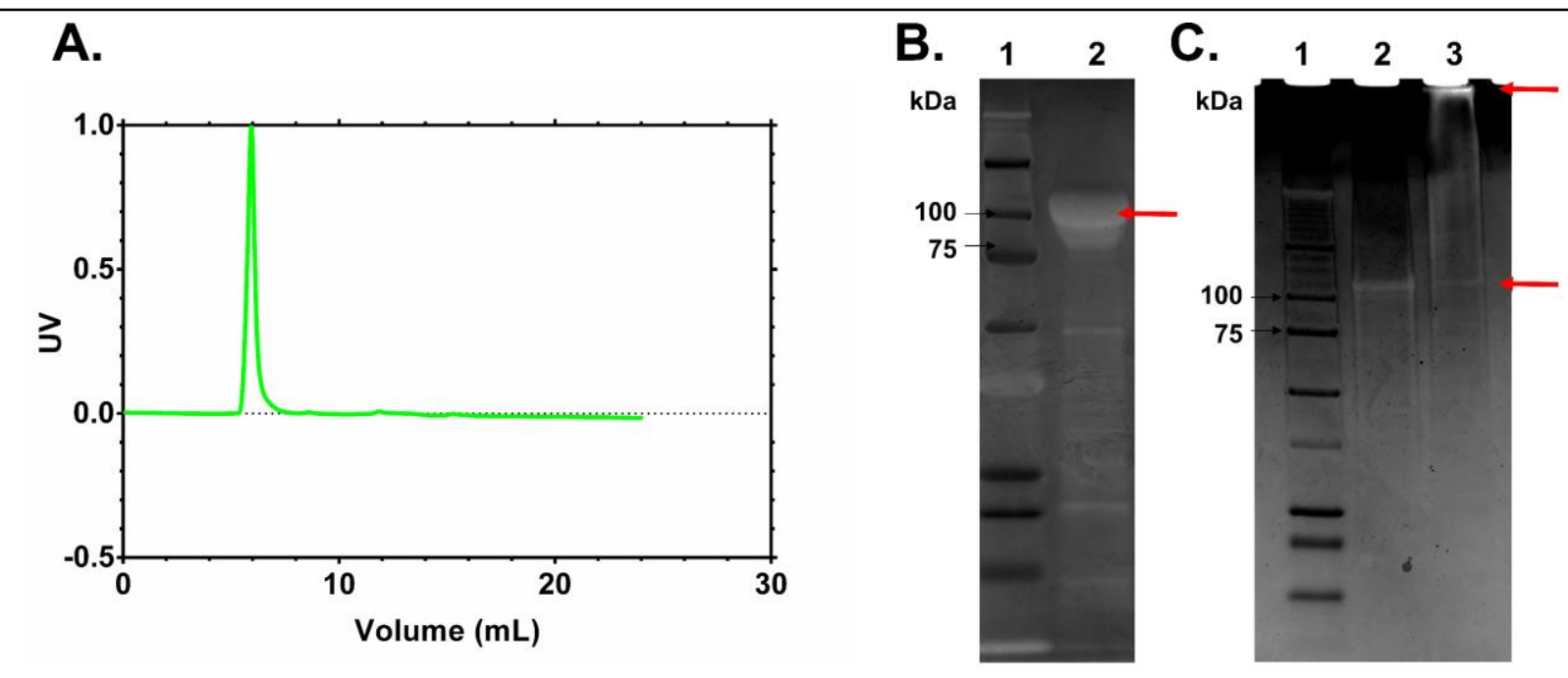

Figure S1. The purity and characteristic of hGMCSF-A192. A) To measure the purity of hGMCSFA192, UV trace of hGMCSF-A192 was obtained from SEC-MALS data. The UV trace showed one major peak and one minor peak, and the AUC of the major peak was calculated to be $99.2 \%$ of the total AUC. B) The gel image shows the purity of the $1^{\text {st }}$ batch of hGMCSF-A192. The gel was stained with copper chloride, the purity of the fusion protein was measured to be $89.2 \%$ using ImageJ. C) The $2^{\text {nd }}$ batch of hGMCSF-A192 was run on an SDS-PAGE gel with (Lane 2) and without (Lane 3) a reducing agent. When the fusion protein was run with a reducing agent, the protein band was located near the $100 \mathrm{kDa}$ marker, and the purity of hGMCSF-A192 was measured to be near $100 \%$ using ImageJ. When the fusion protein was run without a reducing agent, a major portion of hGMCSF-A192 was not able to run on the gel, indicating that a large fraction of hGMCSF-A192 forms disulfide bonds intermolecularly. 